\title{
WORKING TOGETHER: EDUCATION, RESEARCH AND DEVELOPMENT FOR 5G NETWORKS
}

\author{
Ivanova E. P. ${ }^{1}$, Ilev T. B. ${ }^{1}$, Mihaylov Gr.Y. ${ }^{1}$, Stoyanov I.S. ${ }^{1}$, Yehorov V.B. ${ }^{2}$ \\ ${ }^{1}$ University of Ruse "Angel Kanchev" 8 Studentska Str, 7017 Ruse, Bulgaria \\ ${ }^{1}$ E-mail: epivanova@uni-ruse.bg \\ ${ }^{2}$ Research laboratory mechatronics and robotics, Odessa National Academy of Food Technologies (Odessa, Ukraine) \\ ${ }^{2}$ E-mail: yegorov-victor@ mail.ru \\ Copyright (C) 2014 by author and the journal "Automation technological and business - processes". \\ This work is licensed under the Creative Commons Attribution International License (CC BY). \\ http://creativecommons.org/licenses/by/4.0/
}

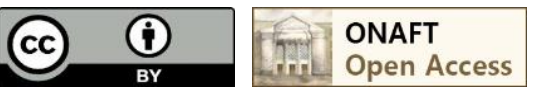

DOI: $10.15673 /$

\begin{abstract}
In the new world of globalization of ideas and mobility difficulties in knowledge diffusion still remains. The effective exchange of experiences and skills in new generation networks is not guaranteed by the enormous potentials ofinternetworking systems and devices. Conceptual model for performance modeling and evaluation of multiservice networks has been major interest for mobile networks providers. It is essential to assess the performance of mobile system architectures in order to identify where potential bottlenecks and data packet blocking probability are possible to occur. Educational platforms, new simulations opportunities represent a good opportunity to reduce the digital divide and to ensure faster and higher communication trends. Several universities and companies are currently involved in using educational platforms to provide better results. Conceptual model for teletraffic engineering in educational platform and applications focuses on some important aspects: tutorials, exercise, simulations, and expectation values of parameters, testing and estimation of students work. In the same time the same model is very appropriate for simulation of network management for the new generation networks. This educational platform for academics, students and researchers, puts together some of the critical aspects of distributed systems and their characteristics, parameters and probability of blocking.
\end{abstract}

Key words

5G, Blocking Probability, Teletraffic Engineering, Queue Model.

\section{INTRODUCTION}

Defining the conceptual model for educational platform for Teletraffic Engineering is one of the main parts in student education. In this case the applications focus on network management for the new generation networks and distributed computing technologies. Besides it presents emerging technology trends and advanced platforms. This educational platform for academics, students and researchers, puts together some of the critical aspects of distributed systems, their characteristics and probability of blocking.

Teletraffic simulation for educational purposes, utilizes complex models to study complicated telecommunication problems. Those models include data modeling for objects, behavior models for interaction, and other algorithms such as randomization, variations, blocking etc. As no unique model fits every scenario, researchers from transportation community are investigating new customable simulation models continuously. This paper, thereby, presents a prototype of a research and education oriented simulation platform, which helps researchers and students to reduce their workload by providing a compact data structure, a friendly interface, and essential automation procedures, with teletraffic simulation $[3,6,9]$. 
ADVANTAGES IN "WORKING TOGETHER"

Teletraffic theory is defined to solve problems with planning, performance evaluation, operation and maintenance of telecommunication systems [5]. First theory was illustrated by examples from telephone networks, but nowadays the students are not interested in such topics. In 5G mobile networks the necessity of traffic measurement and quality of service

must be observed [2]. Working together developers, researchers and students define some critical aspects in network. The idea of the educational development of models, algorithms and simulations make consolidation and exchange of experience of interests in the two groups [1,2].

Research methodology is suggested for students of corporate strategy in order to attain generalizability and statistical significance in reporting findings while not losing the understanding of teletraffic engineering [7]. The advantages for the students are:

- High prestige and technology work;

- Training period is connected with future skills;

- High motivated working area;

- Contacts with partners in all over the world;

- Possibility of professional grows;

- Perspective with long-dependence investment;

- Time for studies, courses and qualification exams;

- Financial rewards.

Research and Development departments are common in many larger companies and universities working with newer products or technologies subject to important shifts [10].

While research and development work can be instrumental in creating new products or adding features to old products, the work that the department does is more complex than simple innovation. R\&D is connected to marketing, cost management and other parts of business strategy.

\section{G TRENDS}

The trends of $5 \mathrm{G}$ mobile access is to be built upon new radio access technologies, evolving 4G, LTE, HSPA, GSM and $\mathrm{Wi}-\mathrm{Fi}$. The new requirements are widely varying. The services, devices, number of clients are in super wide variety.

The new communication needs of a massive deployment of various devices, such as with low complexity parameters to more advanced parameters. The industrial and medicine devices must be lifesaving.

The services must be amazingly fast in very dense crowds of users [1]. The fast connections is a key factor for success of development of so important services as health and security sectors, fire brigade, high speed trains and cars (Fig.1).

The services are:

- Shopping logistic;

- Vehicular telematics;

- Virtual reality;

- Emergency;

- Stadium, crowd of people;

- Wireless cloud offices;

- High speed trains;

- Multiuser UHD telepresence;

- HDTV;

- Mirror Systems;

- Social games;

- 2G, 3G and 4G services;

- Smart sensors. 


\section{ОГЛЯДИ ТА РЕКЛАМНІ МАТЕРІАЛИ}

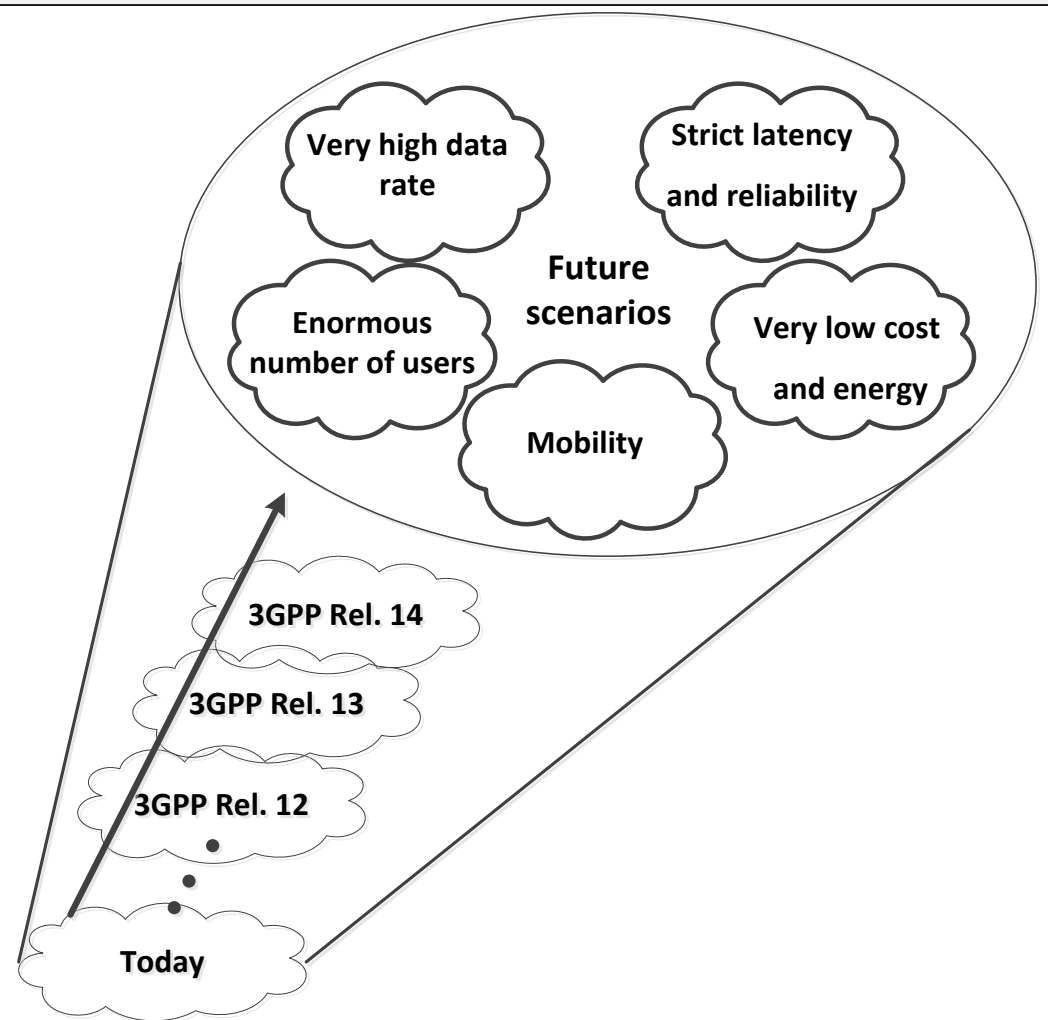

Fig. 1 .Trends of 5G mobile networks

The Quality of Experience requires fully connected users not only for static users. The expectation of connectivity is no matter where or how one is moving [1]. Highly mobile devices, e. g. trains and cars, are communicating machines for this scenario, but also sensors related to widely varying applications, e.g. moving components in industries, plants, other vehicles [8].

Data networks [3,4], broadband networks, Broadband Convergence Networks, ATM, fast IP networks, all generations of mobile secular networks are extremely growing network, in a contrast to the conventional cable systems [9,10,11]. The future engineers must be with very high knowledge in all areas of telecommunications.

\section{REQUIREMENTS FOR SIMULATION PLATFORM}

The main idea of the simulation platform is to cover different aspects of data communications and telecommunications. The future engineers must be with very high knowledge in all areas of telecommunications.

It will be implemented on .NET [12]. Presented work introduces a part of conceptual model of the whole simulation platform, including:

- Educational part;

- Business part;

- Research part.

The model includes modules for education, tests, student's research and programming of different networks. In the past the teletraffic theory was illustrated examples of non-digital telephones and technologies. But nowadays the task is to design systems as cost effectively as possible with predefined quality of experience, grade and quality of service especially, when we know the future traffic demand and the capacity of system elements. Even the most important task is to specify the methods for control, to specify emergency actions when system is overloaded or blocked. This is a necessary step for applying the theory in the practice, a series of decision problems.

Modeling of various telecommunication systems must describe the whole or a part of the system. The requirements of the modeling process for new applications are knowledge of: technical system; mathematical model and implementation of the model. New telecommunication systems must be defined with: system structure, operational strategy and statistical properties of the traffic.

Data networks [11,12], broadband networks, Broadband Convergence Networks, ATM, fast IP networks, all generations 
of mobile secular networks are extremely growing network, in a contrast to the conventional cable systems [1,6,7]. Three simulation teletraffic modules, HeavyQueues, ComplexQueues and HandoverSchemes, ensure a platform for data modeling, simulation flow, and user interfaces, respectively. The nature of the dynamic structure lets users an easy work with the complicated simulation program by extending the default essential data and simulation models and plugging in the partitioned simulation flow.

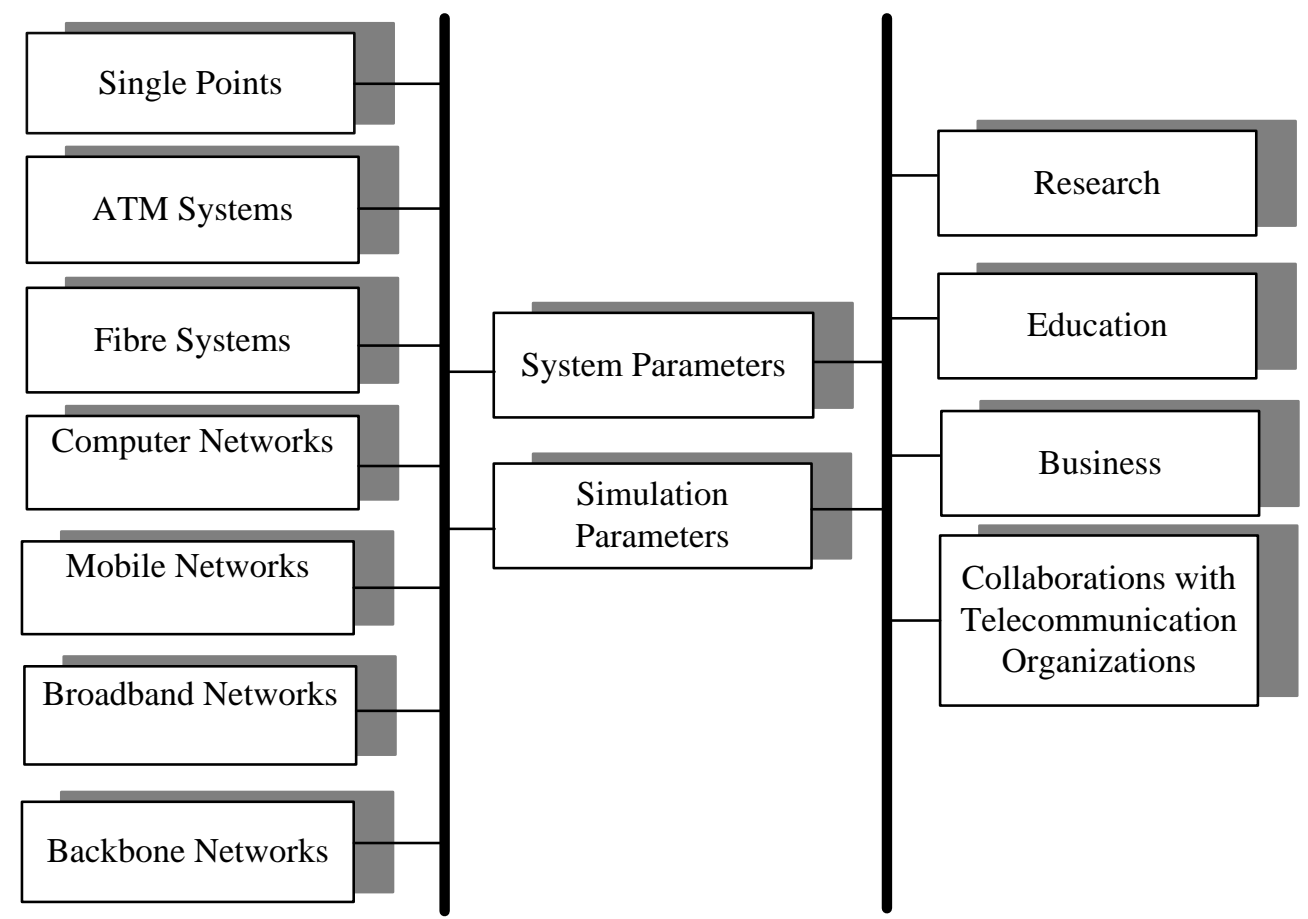

Fig. 2. Simulation platform for data modeling and simulations

The simulation experiments for the next generation mobile networks have to be realized with different teletraffic systems. The realization of this conceptual model will be developed with .NET Framework, which can operate with 15 different programming languages. His main component- Common Language Runtime operate with Visual Basic .NET for realized simulations [12].

The working groups [1] defined $5 \mathrm{G}$ network requirements for simulation as:

- $1 \mathrm{~Gb} / \mathrm{s}$ to be offered, simultaneously to tens of workers on the same office floor;

- Up to Several 100,000's simultaneous connections to be supported for massive sensor deployments;

- Spectral efficiency should be significantly enhanced compared to $4 \mathrm{G}$;

- Coverage should be improved;

- Signaling efficiency enhanced.

Although these requirements seem quite vague they are targeting three foreseen problems for year 2020, which is the aimed time frame for succeeding the development:

- Avalanche of traffic: Explosion of the number of connected devices. From 5 billion (2010) to 50 billion (2020). Keywords are Internet of things, e.g. wearables, housing;

- Large diversity of use cases and requirements: guaranteed service level, reliability ,latency ,device capabilities, Device-to- Device;

- Communications Car-to-Car Comm. rapidly developing Advanced Driver Assistant Systems (ADAS), e.g. Infotainment Systems, Traffic jam assistants, also autonomous driving is aimed as vision for 2020.

For a more detailed view at vehicle to vehicle communication, we need to take a closer look at the requirements to enable this and to work out the obstacles that have to be dealt with. The METIS2020 research group has therefore designed a scenario to visualize the problem and demand for traffic safety and efficiency [9].

The main challenges of this test case lie in the required reliability, availability, and latency of automotive safety services. A maximum network end-to-end delay (including device detection, connection setup and radio transmission) of 5 [ms], with transmission reliability of $99.999 \%$ should be guaranteed to deliver the drive safety service. 


\section{ОГЛЯДИ ТА РЕКЛАМНІ МАТЕРІАЛИ}

Additional key points that have to be taken in consideration are:

- V2X communication needs to be established across different network operators with the same requirements in terms of latency and service guarantee as within a single network operator.

- $100 \%$ availability required such that the services are present at every point on the road;

- Relative positioning accuracy below $0.5 \mathrm{~m}$ is needed. GPS may not always be available and sufficient, and hence cellular based positioning techniques could be useful;

- Periodic and event-driven broadcast traffic consisting of at least 1600 payload [byte] with repetition rate of at least $5-10[\mathrm{~Hz}]$. The update rate is chosen high enough such that the vehicle velocity vector does not change too much between updates. The traffic generated by each vehicle has to be delivered to all the neighboring vehicles within the specified range;

- Both traffic types (periodic and event driven) can exist at the same time;

- For communication between vehicles and other devices (e.g. smartphones) a payload of 500 [byte] may be sufficient (for transmission of the information from the actual consumer electronics device, such as current position and additional data from the device sensors).

\section{CONCLUSION}

Educational platforms, new simulations opportunities represent a good opportunity to reduce the digital divide and to ensure faster and higher communication trends. Several universities and companies are currently involved in development of simulation teletraffic platform to provide better results. The paper presented some of the requirements for development of the simulation teletraffic model not only for educational purposes used in two of the Bulgarian universities and Odessa National Academy:

University of Ruse and University of Telecommunications and Post in Sofia,

Research laboratory mechatronics and robotics, Odessa National Academy of Food Technologies.

References

[1] Brahmi N., METIS: Mobile Communications for 2020 and beyond, VDE/ITG Fachtagung Mobilkommunikation, 2013;

[2] Iliev, T., Gr. Mihaylov. 3GPP LTE system model analysis and simulation of video transmission. Proceedings in Advanced Research in Scientific Areas, Slovak Republic, 2012, pp. 2016-2021;

[3] Ivanova, E. Modeling of the traffic in broadband convergence networks, PhD dissertation, University of Ruse, 2013;

[4] Ivanova E., T. Iliev. Trends in development of next generation networks, Journal of the Union of Scientist Ruse, vol.12, 2015, pp.50-54 (in bulgarian);

[5] Mihaylov Gr., T. Iliev, E. Ivanova. Algorithm for Content Adaptation of Multimedia Information. International Journal of Engineering, Business and Enterprise Applications (IJEBEA), 2015, No 11, pp. 1-7;

[6] Otsetova-Dudin E., Radeva S. Simulation modeling of broadband wireless handover at cellular radio networks.// Automation and Information, 2012, No 5-6, pp. 311-314;

[7] Popovski P., Ultra-Reliable Communication in 5G Wireless Systems, 1st International Conference on 5G for Ubiquitous Connectivity 5 GU Conference, 2014;

[8] Pratas N., P. Popovski, Underlay of Low-Rate Machine-Type D2D Links on Downlink Cellular Links, IEEE International Conference on Communications, ICC 2014, 2014;

[9] Radev D., Teletraffic Engineering, University of Ruse Press, 2012;

[10] Radev D., R. Raev, T. Iliev, Gr. Mihailov, E. Ivanova. Conceptual Model for Educational Platform for Simulation of Teletraffic Engineering. 13th International Conference on Information Technology Based Higher Education and Training - ITHET 2014, York, England, 2014, pp.1-4;

[11] Raev R, Ivanova E, Dudin E, Radev D. Educational Platform For Teletraffic Engeneering, 6th International Conference on Education and New Learning Technologies, Barcelona, Spain, 2014, No 1, pp. 7122-7127;

[12] Sells, C., Windows Forms Programming in C\#, 2003. 